\title{
$\beta$-decay study of ${ }^{77} \mathrm{Cu}$
}

\author{
N. Patronis, ${ }^{1,}{ }^{*}$ H. De Witte,${ }^{1}$ M. Gorska,${ }^{1, \dagger}$ M. Huyse,${ }^{1}$ K. Kruglov, ${ }^{1}$ D. Pauwels,${ }^{1}$ K. Van de Vel, ${ }^{1}$ P. Van Duppen, ${ }^{1}$ \\ J. Van Roosbroeck, ${ }^{1}$ J.-C. Thomas, ${ }^{2}$ S. Franchoo, ${ }^{3, \ddagger}$ J. Cederkall, ${ }^{3}$ V. N. Fedoseyev, ${ }^{3}$ H. Fynbo, ${ }^{3,}$ U. Georg, ${ }^{3}$ O. Jonsson, ${ }^{3}$ \\ U. Köster, ${ }^{3, \|}$ T. Materna, ${ }^{4}$ L. Mathieu, ${ }^{5}$ O. Serot, ${ }^{5}$ L. Weissman, ${ }^{6}$ W. F. Mueller, ${ }^{6}$ V. I. Mishin, ${ }^{7}$ and D. Fedorov ${ }^{8}$ \\ ${ }^{1}$ Instituut voor Kern-en Stralingsfysica, Katholieke Universiteit Leuven, Celestijnenlaan 200D, B-3001 Leuven, Belgium \\ ${ }^{2}$ Grand Accelarateur National d'Ions Lourds (GANIL), CEA/DSM-CNRS/IN2P3, B. P. 55027, F-14076 Caen Cedex 5, France \\ ${ }^{3}$ ISOLDE, CERN, CH-1211, Genève 23, Switzerland \\ ${ }^{4}$ Institut Laue Langevin, 6 rue Jules Horowitz, F-38042 Grenoble Cedex 9, France \\ ${ }^{5}$ CEA-Cadarache, DEN/DER/SPRC/LEPh, F-13108 Saint Paul lez-Durance, France \\ ${ }^{6}$ National Supercontacting Cyclotron Laboratory, Michigan State University, 164 S. Shaw Lane, Michigan 48824-1312, USA \\ ${ }^{7}$ Institute of Spectroscopy, Russian Academy of Sciences, RU-142092 Troitsk, Russia \\ ${ }^{8}$ St. Petersburg Nuclear Physics Institute, RU-188350 Gatchina, Russia \\ (Received 17 June 2009; published 9 September 2009)
}

\begin{abstract}
A $\beta$-decay study of ${ }^{77} \mathrm{Cu}$ has been performed at the ISOLDE mass separator with the aim to deduce its $\beta$-decay properties and to obtain spectroscopic information on ${ }^{77} \mathrm{Zn}$. Neutron-rich copper isotopes were produced by means of proton- or neutron-induced fission reactions on ${ }^{238} \mathrm{U}$. After the production, ${ }^{77} \mathrm{Cu}$ was selectively laser ionized, mass separated, and sent to different detection systems where $\beta-\gamma$ and $\beta-n$ coincidence data were collected. We report on the deduced half-live, decay scheme, and possible spin assignment of ${ }^{77} \mathrm{Cu}$.
\end{abstract}

DOI: 10.1103/PhysRevC.80.034307 PACS number(s): 23.20.Lv, 23.40.-s, 27.50.+e, 21.10.Tg

\section{INTRODUCTION}

Recently, theoretical as well as experimental studies indicate that the size of shell gaps can alter when changing the $N / Z$ ratio leading to changes in magic numbers when going away from the valley of stability [1]. An interesting region of the nuclear chart to study this phenomenon is situated around ${ }^{68} \mathrm{Ni}$ because of the closed proton shell $(Z=28)$ and the closed harmonic-oscillator subshell $(N=40)$. The robustness of the $Z=28$ shell closure as neutrons are added in the unique-parity orbit $\nu g_{9 / 2}$ is of special interest and the induced core-polarization effects have been studied together with the fragile nature of the $N=40$ subshell closure by means of different experimental approaches. Coulomb excitation studies in the neutron-rich $\mathrm{Ni}$ isotopes [2,3] resulted in small $B(E 2)$ values for $N=40$, suggesting a new subshell closure in concert with the high excitation energy of the $2_{1}^{+}$state [4]. On the other hand, Grawe and Lewitowicz [5] and Langanke et al. [6] explained the small observed $B(E 2)$ strength and the high excitation energy of the $2_{1}^{+}$state in a different way. On the same ground, recent mass measurements in this region show a very weak irregularity in the two-neutron-separation energies around $N=40$ giving no evidence for a well-established

\footnotetext{
*Present address: Department of Physics, The University of Ioannina, GR-45110 Ioannina, Greece; npatronis@cc.uoi.gr

${ }^{\dagger}$ Present address: GSI, Planckstrasse 1, D-64291 Darmstadt, Germany.

${ }^{\dagger}$ Present address: Institut de Physique Nucléaire, IN2P3CNRS/Université Paris-Sud, F-91406 Orsay Cedex, France.

${ }^{\S}$ Present address: Department of Physics and Astronomy, University of Århus, DK-8000 Århus, Denmark.

"Present address: Institut Laue Langevin, 6 rue Jules Horowitz, F-38042 Grenoble Cedex 9, France.
}

shell closure $[7,8]$. The same mass region was further studied by means of Coulomb excitation and $\beta$-decay experiments in neighboring $\mathrm{Cu}$ and $\mathrm{Zn}$ neutron-rich isotopes [9-15]. From those studies the fragile nature of the $N=40$ subshell closure was confirmed and the key role of the $g_{9 / 2}$ orbit was revealed in the systematics. A common conclusion in the above-mentioned surveys is the need for further spectroscopic information in this region and especially toward the more exotic nuclei around the doubly magic ${ }^{78} \mathrm{Ni}$.

Another interest for the region around $Z=28$ and $40<$ $N<50$ is related to the astrophysical rapid neutron-capture nucleosynthesis process, i.e., the $r$ process. The $r$ process is responsible for the origin of about half of the elements heavier than iron. Nuclei with closed neutron shells represent a special set of waiting points that cause a vertical diversion of the r-process path in the neutron-rich side of the chart of nuclei. ${ }^{78} \mathrm{Ni}$ is a doubly magic nucleus that represents the most important waiting point of the $r$ process [16]. For this reason the $\beta$-decay properties of the nuclei in the region as well as the spectroscopic information are of paramount importance in r-process calculations.

In this article we report on the $\beta$-decay study of ${ }^{77} \mathrm{Cu}$. This study is part of a systematic $\beta$-decay study at ISOLDE, CERN [17], of neutron-rich $\mathrm{Cu}$ isotopes ranging from ${ }^{71} \mathrm{Cu}$ to ${ }^{78} \mathrm{Cu}$. The odd-odd cases are described in Refs. [10,18-21]. The results on the odd-mass ${ }^{71-75} \mathrm{Cu}$ are discussed in the Ph.D. work of Faul [22]. The lowering of the first-excited states with spin and parities $5 / 2^{-}$and $1 / 2^{-}$, as the neutron number increases in odd copper isotopes with $A>69$ [9], makes particularly ${ }^{77} \mathrm{Cu}$ an interesting physics case. More specifically the lowering of the $5 / 2^{-}$relative to the $3 / 2^{-}$state is expected to be strong enough to have a $5 / 2^{-}$ground state in ${ }^{77} \mathrm{Cu}$.

In the following, we discuss the experimental setup used (Sec. II) along with the obtained results (Sec. III). In the last 


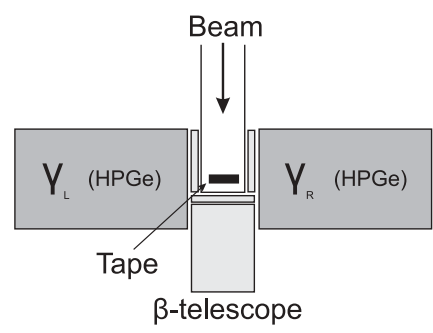

FIG. 1. Top view of the detector geometry at the $\beta$-decay setup. The $\beta$ detectors are indicated with the light gray color.

section the proposed ground-state spin and parity of ${ }^{77} \mathrm{Cu}$ is discussed. In the same section a short interpretation is given for the spin and parity assignments of the ground state and low-lying excited states of ${ }^{77} \mathrm{Zn}$.

\section{EXPERIMENTAL SETEUP}

The neutron-rich copper isotopes were produced via protonand neutron-induced fission of ${ }^{238} U$ target. More specifically the $1 \mathrm{GeV}$ proton beam delivered from the CERN PS-Booster either impinged directly on the thick $50 \mathrm{~g} / \mathrm{cm}^{2}$ uranium carbide target or was sent to a tantalum proton-to-neutron converter to induce fission through the produced spallation neutrons. For the case of ${ }^{77} \mathrm{Cu}$, the latter technique was used to suppress the $\mathrm{Rb}$ isobaric contamination, produced through spallation and present in the mass-separated beam because the surface ionization of this element is not negligible. Another element that is easily surface ionized is Ga. The Ga isotopes are strongly populated by spallation-neutron-induced fission and thus remain present in the mass-separated beam. The ${ }^{77} \mathrm{Cu}$ nuclei were selectively ionized by means of the resonant ionization laser ion source (RILIS) [23-25] and mass separated by the general purpose separator (GPS) of the ISOLDE facility [17].

After mass separation, the radioactive beam with a typical energy of $60 \mathrm{keV}$ was delivered to the $\beta$-decay experimental setup (Fig. 1). The ${ }^{77} \mathrm{Cu}$ nuclei were implanted on a tape that was surrounded by three plastic $\Delta E$ detectors for the $\beta$-particle detection. The emitted $\gamma$ rays were recorded through two HPGe detectors with relative efficiencies $75 \%$ (left) and $90 \%$ (right). These two detectors were placed in close geometry, typically at $2 \mathrm{~cm}$ distance, aiming for the maximum possible detection efficiency. The full energy of the $\beta$ particles was recorded with the $\beta$ telescope that was installed behind the implantation tape at $0^{\circ}$ with respect to the beam direction.

The electronics readout was done in parallel by two different methods: the singles mode, where the energy signals of the two $\gamma$ detectors were recorded together with the $E$ part of the $\beta$ telescope, and the list mode data (LMD) acquisition mode, where the data were recorded in an event by event mode. In the LMD mode, the trigger was created whenever a $\beta-\gamma$, a $\beta-\beta$, or a $\gamma-\gamma$ event was recorded. Given the much higher efficiency of the $\beta$-detection setup most of the LMD data correspond to $\beta$-triggered events.

For a more precise lifetime determination the ${ }^{77} \mathrm{Cu}$ beam was sent to a second setup to detect the emitted $\beta$-delayed

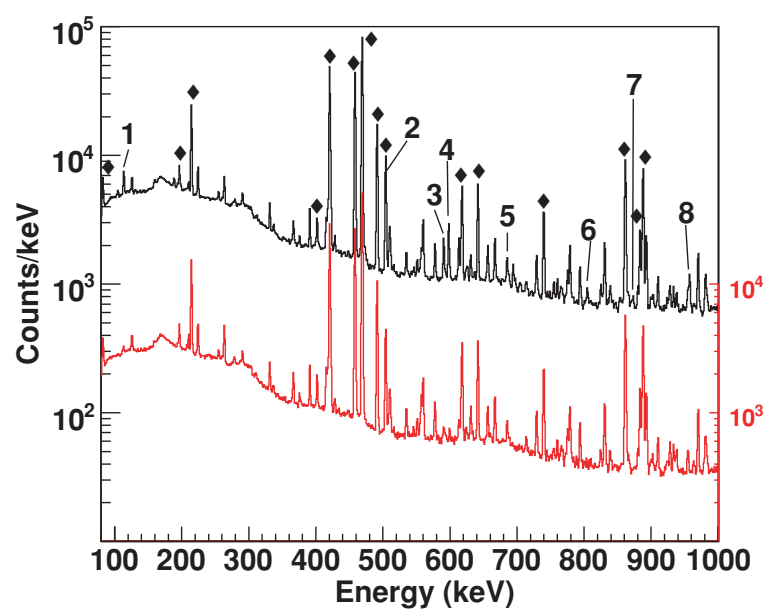

FIG. 2. (Color online) Part of the LMD-based lasers-on (upper black histogram) and lasers-off (lower red histogram) spectra. Lines labeled with a number belong to the ${ }^{77} \mathrm{Cu}$ decay and are listed in Table II where the intensity is given. The transitions indicated with $\checkmark$ correspond to the decay of the surface-ionized ${ }^{77} \mathrm{Ga}$ contaminant.

neutrons. It consisted of the Mainz neutron long counter [26] with a built-in tape transport system to remove long-lived background after each measurement cycle. A thin plastic scintillator served for the detection of $\beta$ particles. Despite the selectivity of the RILIS on mass 77 the $\beta$ activity was dominated by gallium. Therefore the $\beta$ rays of the ${ }^{77} \mathrm{Cu}$ decay could not be discriminated and no $P_{n}$ value could be deduced. Nevertheless, the $\beta$-delayed neutron signal was very clean because the isobaric contaminants do not emit neutrons. Measurements with the RILIS lasers off or detuned from the resonance gave only a flat background (cosmic radiation).

The timing signals of the neutron counters were fed into an eight-input multichannel scaler (module 7884 from Fast Comtec) that can handle data rates above $1 \mathrm{MHz}$. It was read out by a MPA2 DAQ from Fast Comtec. For calibration, the entire acquisition system was tested at up to two orders of magnitude higher data rates (abundant $\mathrm{Rb}$ and $\mathrm{Cs}$ isotopes) but showed no significant dead time effects.

In Table I, a summary of the experimental conditions for the decay study of ${ }^{77} \mathrm{Cu}$ is given. Each run is simply a repetition of cycles, where each cycle has a characteristic implantation and decay period depending on the half-life of the isotope under study. After such a cycle, the tape is moved to suppress the long-lived background activity. For each isotope, a certain amount of beam time was used without using the lasers (lasersoff runs) to determine the beam contamination (mainly $\mathrm{Ga}$ ) and the room background. A more detailed description of the experimental setup can be found in Refs. [15,18,19].

TABLE I. Experimental conditions for the decay study of ${ }^{77} \mathrm{Cu}$.

\begin{tabular}{lcc}
\hline \hline $\begin{array}{l}\text { Cycle } \\
\text { implantation/decay time }\end{array}$ & $\begin{array}{c}\text { Laser on } \\
\text { measurement time }\end{array}$ & $\begin{array}{c}\text { Laser off } \\
\text { measurement time }\end{array}$ \\
\hline $0.6 \mathrm{~s} / 2.2 \mathrm{~s}$ & $170 \mathrm{~min}$ & $115 \mathrm{~min}$ \\
\hline \hline
\end{tabular}



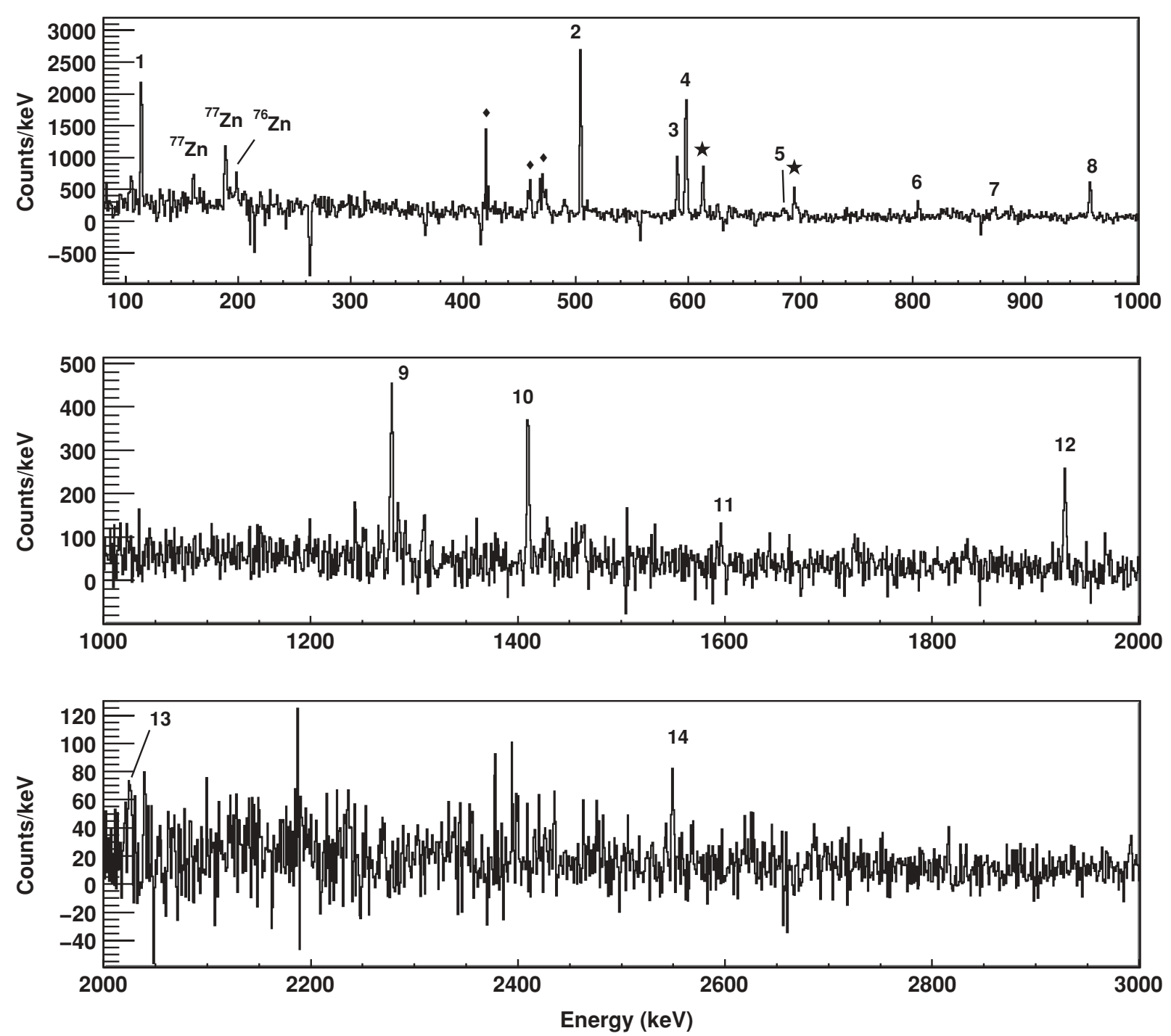

FIG. 3. The LMD-based spectrum after the subtraction of lasers-on minus lasers-off spectra shown in Fig. 2. In this subtracted spectrum the activity coming from the $\beta$ decay of ${ }^{77} \mathrm{Cu}$ can be easily identified (labeled by numbers). The explanation for the negative spikes is given in the text. Also some of the strongest ${ }^{77} \mathrm{Ga}$ lines $(\checkmark)$ are present in the subtracted spectrum, along with some long-lived activity of a previous implantation, ${ }^{78} \mathrm{As}(\star)$.

\section{RESULTS}

The knowledge about the $\beta$-decay properties of ${ }^{77} \mathrm{Cu}$ is limited. The half-life has been determined to be $469 \pm 8 \mathrm{~ms}$ in Ref. [27] and $450_{-21}^{+13} \mathrm{~ms}$ in Ref. [28]. Concerning the levels of the daughter nucleus ${ }^{77} \mathrm{Zn}$ only a few of them have so far been identified. The $772 \mathrm{keV}\left(1 / 2^{-}\right.$isomeric state, $T_{1 / 2}=1.05 \pm$ $0.10 \mathrm{~s}$ ) has been identified in Ref. [29] and the $114.7 \mathrm{keV}$ level together with the $800 \mathrm{keV}$ level has been identified in the $\beta$-delayed neutron emission of ${ }^{78} \mathrm{Cu}[15]$. Only recently, more levels were identified in a decay study at HRIBF [30,31].

The data set for ${ }^{77} \mathrm{Cu}$ can be divided into two parts: the data acquired with the lasers-on resonance and the data acquired with the lasers-off resonance. In Fig. 2 the main part of the LMD-based data using both HPGe detectors is presented. Both the lasers-on and lasers-off spectra correspond to the data recorded during the first $1575 \mathrm{~ms}$ after the starting of the implantation period. In this way almost the full statistics of the decay lines from the short-lived ${ }^{77} \mathrm{Cu}$ decay are obtained while the intensity of the lines corresponding to the long-lived beam contaminants is reduced. As can be seen in Fig. 2 the great majority of the transitions observed are nonresonant, mainly from the decay of the surface-ionized ${ }^{77} \mathrm{Ga}$. The decay scheme of ${ }^{77} \mathrm{Ga}$ is not very well known [32] but the identification of the $\gamma$-ray lines from the $\beta$ decay was feasible given its prominent presence in both on-resonance and off-resonance spectra. The most intense lines related to ${ }^{77} \mathrm{Ga}$ decay are indicated with a diamond symbol $(\diamond)$. Other lines that appear both in the lasers-on and lasers-off spectra are from the $\beta$ decay of long-lived daughter nuclei originating mainly from ${ }^{77} \mathrm{Ga}$ or from other isotopes deposited in the tape system during previous runs associated with different mass settings. As can be seen in Fig. 3 the laser-enhanced lines can easily be identified after normalizing the two spectra of Fig. 2 and subtracting them. The resulting spectrum contains only the peaks related to the ${ }^{77} \mathrm{Cu}$ decay and daughter activity $\left({ }^{77} \mathrm{Zn}\right)$. At this point 


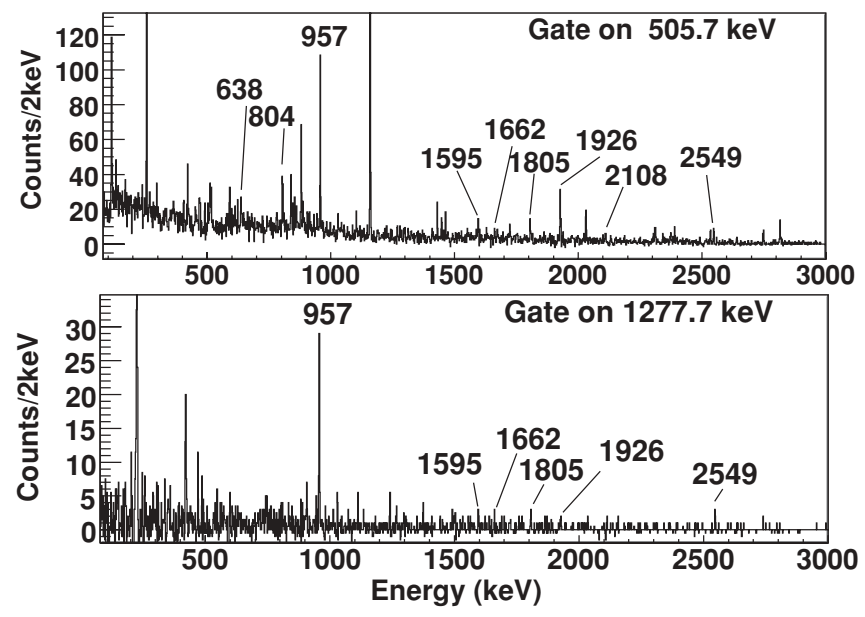

FIG. 4. The $\gamma$-ray spectrum of prompt coincidence events with $506 \mathrm{keV}$ (upper panel) and $1278 \mathrm{keV}$ (lower panel) lines. Only the lines from ${ }^{77} \mathrm{Cu}$ decay are labeled. Unlabeled lines come from ${ }^{77} \mathrm{Ga}$ contamination.

it is worth noting that even in the subtracted spectrum the strongest ${ }^{77} \mathrm{Ga}$ lines are still present. The negative counts are due to long-living ${ }^{77} \mathrm{Ge}(11.3 \mathrm{~h})$ which has been growing in since the measurements started. Given the fact that the measurement started with the lasers-on resonance and then later with lasers off, relatively more counts of ${ }^{77} \mathrm{Ge}$ are present in the lasers-off spectrum. The $772 \mathrm{keV}$ transition is not present in the coincidence spectra because of the long half-life time $\left(\sim 1 \mathrm{~s}\right.$ [29]) of the isomeric state. Because the decay of ${ }^{77} \mathrm{Zn}$ is well known [32] the identification of resonant lines attributed to the ${ }^{77} \mathrm{Cu}$ decay or to its daughter ${ }^{77} \mathrm{Zn}$ was feasible. In Figs. 2 and 3 the lines assigned to the $\beta$ decay of ${ }^{77} \mathrm{Cu}$ are labeled with a number.

Information on the observed ${ }^{77} \mathrm{Cu}$ decay lines is summarized in Table II. The rather high uncertainty of a number

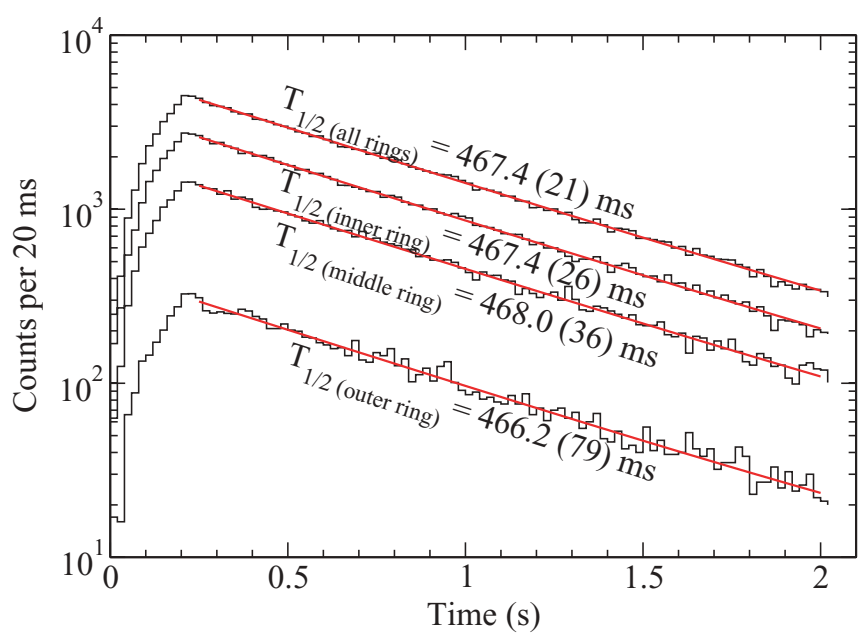

FIG. 5. (Color online) The counting rate of the Mainz neutron long counter during the implantation/decay cycle. The total rate together with the individual rate of each ring is presented with the corresponding exponential fits.

of intensities reflects the fact that many of the lines were contaminated. In these cases, the uncertainty of the resulted peak area is much higher than in the cases of single isolated peaks - free of contaminants (e.g., the $1409.3 \mathrm{keV}$ line). As a second step of control of the $\gamma$-ray assignment procedure, the net area of each transition in the subtracted spectrum was determined by setting different conditions on the absolute time of each coincident event with respect to the proton pulse of the primary beam. In this way for all the cases reported in Table II the time evolution of the net area counts was determined and was found to be compatible with the expected half-life of ${ }^{77} \mathrm{Cu}$. The coincidence $\gamma$ rays were derived from background-subtracted $\gamma$-ray gated spectra with a prompt time gate of $0.6 \mu$ s. In Fig. 4, two coincident $\gamma$-ray spectra are

TABLE II. Energy values and relative intensities of the ${ }^{77} \mathrm{Cu}$ decay lines in the LMD-based spectrum. Coincident $\gamma$ rays are given in the last column. Intensities are indicated within brackets.

\begin{tabular}{|c|c|c|c|}
\hline Label & Energy $(\mathrm{keV})$ & Intensity & Coincident $\gamma$ rays \\
\hline 1 & $114.7(0.3)$ & $33(4)$ & $685.5(4 \pm 1), 1169.8(6 \pm 1)$ \\
\hline 2 & $505.7(0.9)$ & $100(5)$ & $\begin{array}{c}638.0(7 \pm 2), 804.5(16 \pm 2), 957.3(40 \pm 3), 1595.3(8 \pm 2), 1662.1(7 \pm 2) \\
1805.0(8 \pm 2), 1926.0(33 \pm 2), 2108.0(6 \pm 2), 2549(13 \pm 2)\end{array}$ \\
\hline 3 & $591.1(0.2)$ & $34(4)$ & $871.9(10 \pm 2), 1290.8(17 \pm 3), 1840.6(5 \pm 2), 2023.0(13 \pm 2), 2463.6(6 \pm 2)$ \\
\hline 4 & $598.3(0.3)^{\mathrm{a}}$ & $89(3)$ & $698.0(16 \pm 2)$ \\
\hline 5 & $685.5(0.5)$ & $4(1)$ & $114.7(33 \pm 4)$ \\
\hline 6 & $804.5(1.0)$ & $16(2)$ & $505.7(100 \pm 5), 1277.7(46 \pm 4), 1662.1(7 \pm 2)$ \\
\hline 7 & $871.9(1.0)$ & $10(2)$ & $591.1(34 \pm 4)$ \\
\hline 8 & $957.3(0.3)$ & $40(3)$ & $505.7(100 \pm 5), 638.0(7 \pm 2), 1277.7(46 \pm 2)$ \\
\hline 9 & $1277.7(0.2)$ & $46(4)$ & $957.3(40 \pm 3), 1595.3(8 \pm 2), 1662.1(7 \pm 2), 1805.0(8 \pm 2), 1926.0(33 \pm 2), 2549.0(13 \pm 2)$ \\
\hline 10 & 1409.3(0.3) & $37(3)$ & $1463.7(6 \pm 2), 2335.0(7 \pm 3)$ \\
\hline 11 & $1595.3(1.0)$ & $8(2)$ & $505.7(100 \pm 5)$ \\
\hline 12 & $1926.0(0.5)$ & $33(2)$ & $505.7(100 \pm 5), 1277.7(46 \pm 4)$ \\
\hline 13 & $2023.0(1.0)$ & $13(2)$ & $591.1(34 \pm 4)$ \\
\hline 14 & $2549.0(1.0)$ & $13(2)$ & $505.7(100 \pm 5), 1277.7(46 \pm 4)$ \\
\hline 15 & $3826.7(1.0)$ & $3(1)$ & - \\
\hline
\end{tabular}

a $\beta$-delayed neutron emission. 


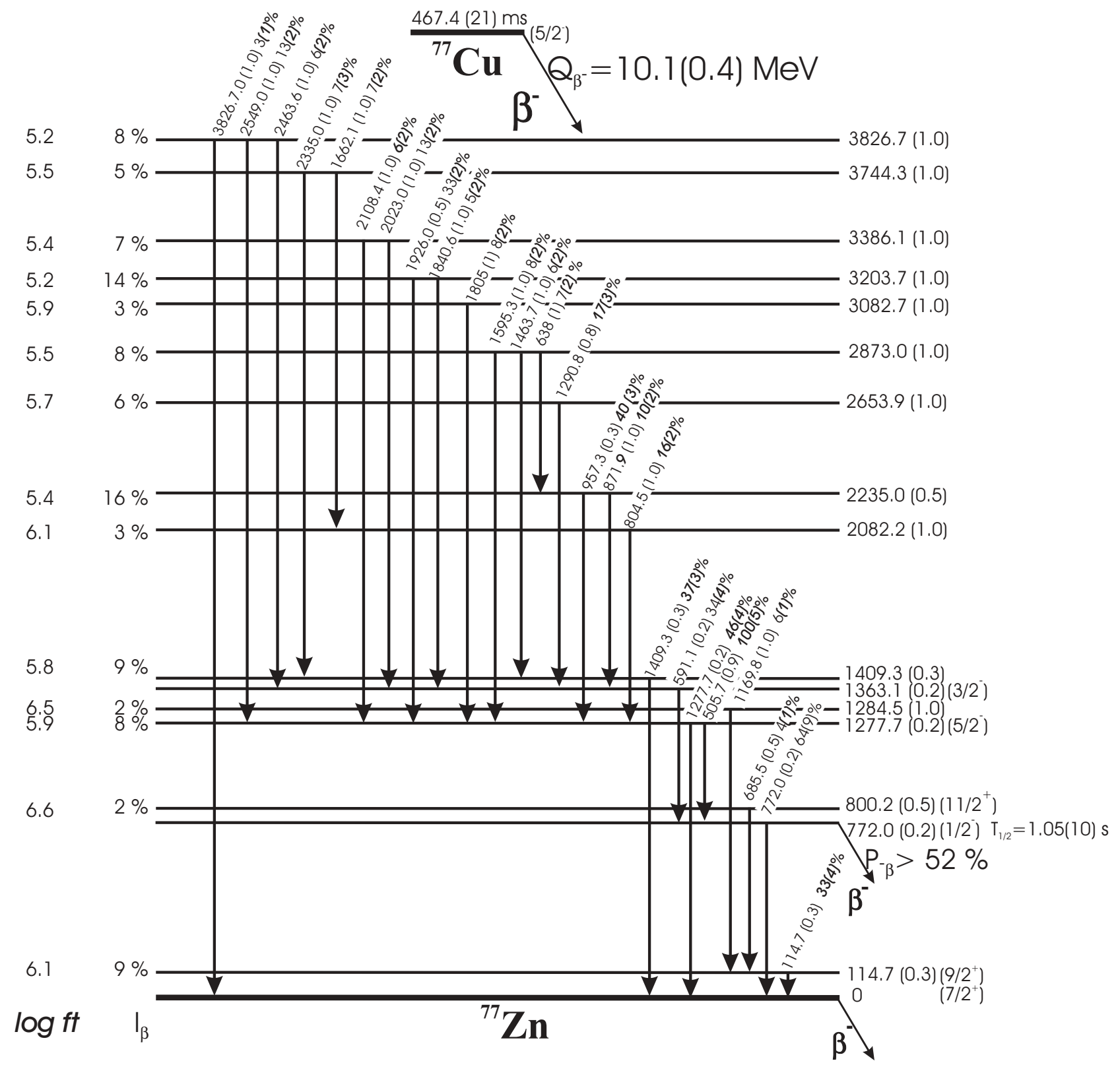

FIG. 6. Deduced decay scheme for ${ }^{77} \mathrm{Cu}$. The $\mathrm{Q}_{\beta^{-}}$value was taken from Ref. [34]. The proposed spin assignments are discussed in the text. The given $\beta$-branching values should be considered only as upper limits and the log $\mathrm{ft}$ values as lower limits.

presented. As can be seen in both spectra, several lines are coming from the ${ }^{77} \mathrm{Ga}$ activity-especially for the $505.7 \mathrm{keV}$ case, which is a doublet. These background lines could be identified because they are also present in the laser-off spectra generated with the same gate conditions.

Because most of the lines were contaminated with other lines, an accurate half-life could only be deduced from the time behavior of two lines: the $957 \mathrm{keV}$ line and the $1409 \mathrm{keV}$ line. The resulting half-life $\left(T_{1 / 2}=467 \pm 43 \mathrm{~ms}\right)$ is less precise than but consistent with the reported values in Refs. [27,28] and with our value out of the $\beta$-delayed neutron measurement. As can be seen in Fig. 5, a single exponential fit of the neutron-counting rate gives a half-life of 467.4(21) $\mathrm{ms}$ for
${ }^{77} \mathrm{Cu}$, consistent with, but far more precise than, the published values $[27,28]$. The latter value will be further used.

The Mainz neutron long counter consists of three concentric rings of ${ }^{3} \mathrm{He}$ tubes. Low-energy neutrons are most likely detected in the innermost ring; higher-energy neutrons cover a larger distance before being moderated and detected and are therefore more likely detected in the middle or outer ring. The so-called "ring ratios," i.e., the relative rate of neutrons detected in the middle to inner or outer to inner ring allow one to conclude on the average energy of the $\beta$-delayed neutrons [33]. The energy-dependent detection efficiency was simulated with the Monte Carlo neutron transport code MCNP. The simulations were validated with well-known emitters of 
$\beta$-delayed neutrons. Thus, from the ring ratio we found an average neutron energy of about $450(150) \mathrm{keV}$ for ${ }^{77} \mathrm{Cu}$ decay.

With a $Q_{\beta}$ value of $10.1(4) \mathrm{MeV}$ for ${ }^{77} \mathrm{Cu}$ from the Audi-Wapstra systematics [34], a neutron binding energy of $4.557 \mathrm{MeV}$ for ${ }^{77} \mathrm{Zn}$ [35], and apparent feedings of $21.6 \%$ and $4.7 \%$, respectively, to the $2_{1}^{+}$and $4_{1}^{+}$states in ${ }^{76} \mathrm{Zn}$, we deduce apparent $\log \mathrm{ft}$ values of 4.1(2) and 4.4(2) to the neutron emitting states populating by neutron emission the $2_{1}^{+}$and $4_{1}^{+}$ states in ${ }^{76} \mathrm{Zn}$. Note that frequently several neutron emitting levels populate the same level in the $A-1$ daughter. Therefore the given $\log \mathrm{ft}$ values are cumulative over all neutron emitting states populating the $2_{1}^{+}$and $4_{1}^{+}$states in ${ }^{76} \mathrm{Zn}$. The uncertainty of the $\log \mathrm{ft}$ values is dominated by the uncertainty in the $Q_{\beta}$ value of ${ }^{77} \mathrm{Cu}$.

The decay scheme for ${ }^{77} \mathrm{Cu}$ shown in Fig. 6 could be constructed based on the coincidence relations given in Table II. Furthermore, use has been made of the $\beta$-delayed neutron-decay data from ${ }^{78} \mathrm{Cu}$ [15] (see also Fig. 7). In this decay, $\gamma$ rays of 115 and $686 \mathrm{keV}$ were seen in cascade and are also observed here in the $\beta$ decay of ${ }^{77} \mathrm{Cu}$. The strong $115 \mathrm{keV}$ line is only coincident with the 686 and $1170 \mathrm{keV}$ lines. The three lines have no connection with other ${ }^{77} \mathrm{Cu}$ decay transitions. In principle, as long as no connecting transitions are found, the $115 \mathrm{keV}$ line could be feeding the $\left(1 / 2^{-}\right)$ isomeric level at $772 \mathrm{keV}$ instead of the ground state as proposed in Ref. [15] and also assumed here. The present level scheme is in general agreement with the partial level scheme in Refs. [30,31] but extends it considerably.

The relative $\gamma$-ray intensities were calculated from the LMD-based data taking into account the variation of the detection efficiency with respect to the $\gamma$-ray energy. Where necessary, the lines were corrected for contaminating $\gamma$ rays using the off-resonance data. The relative intensities were calculated with respect to the most intense line at $505.7 \mathrm{keV}$ and are given in Table II. The close detection geometry can, depending on the multiplicity of the $\gamma$-decay cascade, lead to summing distortions in the intensities. Because only relative $\gamma$-ray intensities are given and the quoted intensity uncertainties are rather high, these distortions can be neglected. Concerning the isomeric $772.0 \mathrm{keV}$ line, the observed counts were corrected for the mother-daughter decay relation taking into account the implantation and decay cycle. For those transitions observed only in the $\gamma-\gamma$ coincidence spectra, coincidence relations and comparison with other lines were used to extract the intensities.

The $\beta$ feeding shown in Fig. 6 was estimated from the intensities given in Table II assuming the feeding to the ground state and to the isomeric state at $772 \mathrm{keV}$ was equal to zero. The same was assumed for the $1363 \mathrm{keV}$ level because the intensity of the $591 \mathrm{keV} \gamma$ line de-exciting this level is within the error-bars equal to the total intensity of the $\gamma$ rays that feed this level. The experimental determination of the possible existence of $\beta$ feeding to the ground state and to the isomeric state was impossible because no single $\beta$ spectrum was acquired that could be compared with the $\beta-\gamma$ coincidence spectrum. Therefore, and also because of unobserved $\gamma$-ray feeding, the given $\beta$-branching values should be considered as upper limits and the $\log f t$ values as lower limits. The quoted $Q_{\beta}$ value was taken from Ref. [34]. Based on the assumption that there is no direct $\beta$ feeding to the $\left(1 / 2^{-}\right)$state at $772 \mathrm{keV}$, a lower limit of $52 \%$ for the $\beta$-decay probability of this state can be deduced on the basis of the balance of feeding and de-excitation $\gamma$-ray intensities. A remarkable feature of the ${ }^{77} \mathrm{Cu}$ decay scheme is the fact that the $\gamma$ rays de-exciting the high-energy levels are feeding a limited number of excited states avoiding intense feeding of the $\left(1 / 2^{-}\right)$isomeric state or the $\left(7 / 2^{+}\right)$ground state. A similar pattern is observed in the decay of ${ }^{75} \mathrm{Cu}$ and ${ }^{73} \mathrm{Cu}$ [22].

In Fig. 7, a partial decay scheme of ${ }^{78,77} \mathrm{Cu}$ is presented with emphasis on the $\beta$-delayed neutron-emission branch. The strong feeding of the $4^{+}$level and especially the $2^{+}$level in ${ }^{76} \mathrm{Zn}$ in the $\beta$-delayed neutron emission of ${ }^{77} \mathrm{Cu}$ indicates that one or more ${ }^{77} \mathrm{Zn}$ high-energy levels above the neutron binding

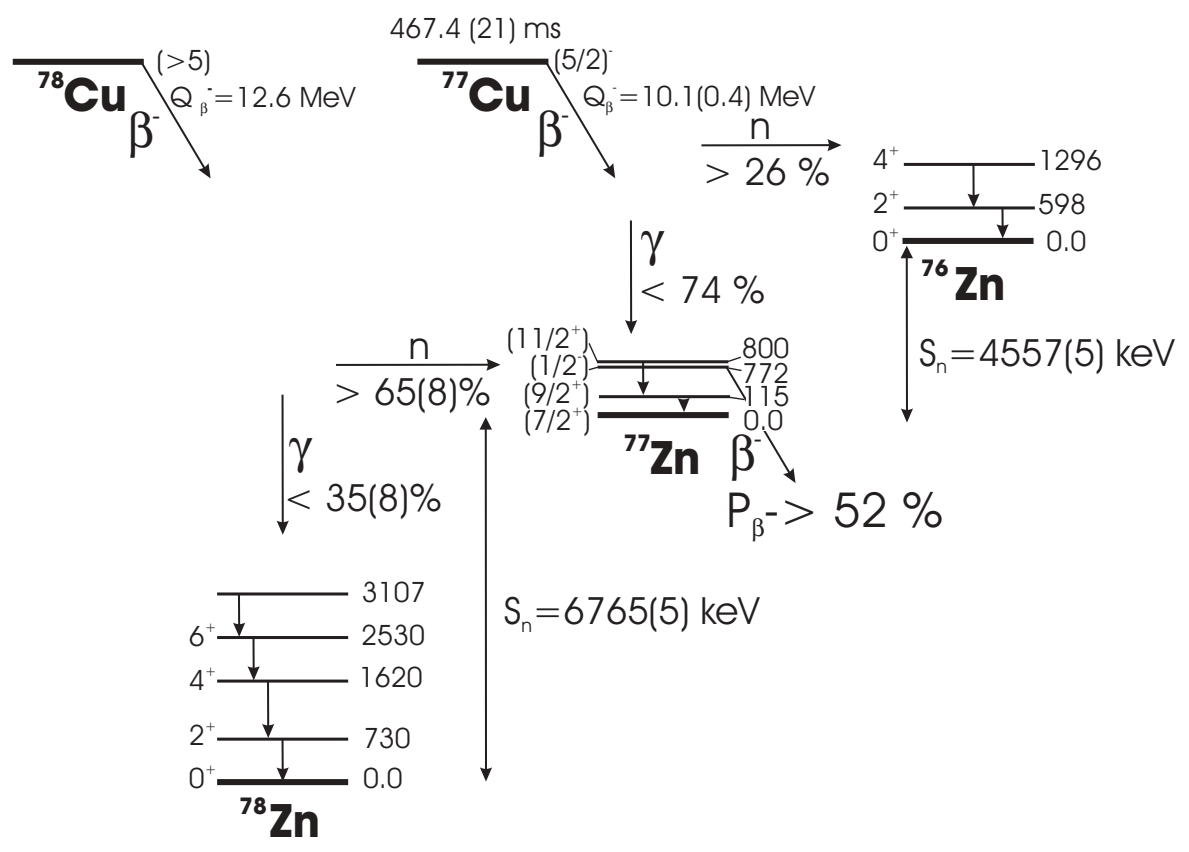

FIG. 7. Abbreviated decay scheme of ${ }^{78,77} \mathrm{Cu}$ with emphasis on the $\beta$-delayed neutron emission branch. Information on the $\beta$-delayed neutron decay of ${ }^{78} \mathrm{Cu}$ is taken from Refs. [15] and [31]. Neutron binding energies are derived from Ref. [35]. 
energy get a large fraction of the overall $\beta$ branching. From the intensity balance of the $598.3 \mathrm{keV} \gamma$ ray and the ${ }^{77} \mathrm{Zn} \gamma$ rays, a lower limit for the probability for neutron emission in ${ }^{77} \mathrm{Cu}$ was obtained: $P_{n} \geqslant 26 \%$, in agreement with the recent results of Winger et al. [31] and much higher than reported in Ref. [36]. Taking into account the spin and energy selection rules for $\beta$ decay and neutron emission and the fact that the $\beta$-delayed neutron channel relatively populates for $18 \%$ the $4^{+}$state and for $82 \%$ the $2^{+}$state in ${ }^{76} \mathrm{Zn}$ limits the possible spin for the ${ }^{77} \mathrm{Cu}$ ground state to $5 / 2$ and $7 / 2$. Combining this information with the fact that the $\beta$-delayed $\gamma$ emission of ${ }^{77} \mathrm{Cu}$ does feed levels that finally end up for relatively $54 \%$ on the $\left(1 / 2^{-}\right)$isomeric state and for $46 \%$ on the $\left(7 / 2^{+}\right)$ground state favors a $5 / 2^{-}$assignment for the ground state of ${ }^{77} \mathrm{Cu}$. In this relative feeding, the $772 \mathrm{keV}$ isomeric transition from the $\left(1 / 2^{-}\right)$isomeric state to the $\left(7 / 2^{+}\right)$ground state is not taken into account. The proposition for a $5 / 2^{-}$spin and parity of ${ }^{77} \mathrm{Cu}$ is in agreement with recent results from laser spectroscopy experiments at ISOLDE where a $3 / 2^{-}$assignment could be excluded leaving only $5 / 2$ or $7 / 2$ as possible spins [37]. In Ref. [30], the $\beta$-decay study of ${ }^{78} \mathrm{Cu}$ seems to point to a feeding pattern different than that observed in our study of ${ }^{78} \mathrm{Cu}$ [15] where the $910 \mathrm{keV}^{+} \rightarrow 4^{+}$transition in ${ }^{78} \mathrm{Zn}$ was not seen. Reanalysis of the data confirms the decay scheme of Ref. [30] (see also Fig. 7) through the observation of the $576 \mathrm{keV}$ line de-exciting the $3107 \mathrm{keV}$ level with an intensity of $17 \pm 10 \%$ relative to the $730 \mathrm{keV}^{+} \rightarrow 0^{+}$transition and a weak peak at $910 \mathrm{keV}$ line $\left(6^{+} \rightarrow 4^{+}\right.$transition $)$with a relative intensity of $12 \pm 9 \%$. The observation of the $6^{+}$level, fed in the decay of ${ }^{78} \mathrm{Cu}$, suggests a spin of at least 5 for the ground state of ${ }^{78} \mathrm{Cu}$. This high spin makes it unlikely that the $\gamma$ transitions observed in the $\beta$-delayed neutron emission of ${ }^{78} \mathrm{Cu}(115 \mathrm{keV}, 686 \mathrm{keV})$ can be placed on top of the $\left(1 / 2^{-}\right)$isomer in ${ }^{77} \mathrm{Zn}$ but rather are connected to the ground state $\left(7 / 2^{+}\right)$. The spin and parity suggestion of $\left(9 / 2^{+}\right),\left(11 / 2^{+}\right)$of, respectively, the $115 \mathrm{keV}$, $800 \mathrm{keV}$ levels is based on the feeding pattern observed in the ${ }^{77,78} \mathrm{Cu}$ decay. The present decay scheme of ${ }^{77} \mathrm{Cu}$ is certainly not complete because the relative strong population of the low-lying positive parity states cannot result from direct $\beta$ feeding from the $\left(5 / 2^{-}\right){ }^{77} \mathrm{Cu}$ ground state. Two explanations are possible: missed $\gamma$-ray feeding from higher-lying levels or the existence of a $\beta$-decaying isomer in ${ }^{77} \mathrm{Cu}$. The latter can probably be excluded because there is no evidence seen in the laser spectroscopy studies of ${ }^{77} \mathrm{Cu}$ [37].

\section{DISCUSSION}

According to the results of the present work, a 5/2spin assignment is proposed for the ground state of ${ }^{77} \mathrm{Cu}$. In Fig. 8, the systematics of the $1 / 2^{-}, 5 / 2^{-}$, and $3 / 2^{-}$states that correspond to the ground and first excited states of the neutron-rich odd $\mathrm{Cu}$ isotopes are presented. As can be seen in this figure for ${ }^{63-69} \mathrm{Cu}$, the position of the first $5 / 2^{-}$level lies almost constantly $1 \mathrm{MeV}$ above the $3 / 2^{-}$ground state. However, when the $v g_{9 / 2}$ neutron orbital starts to be filled, the gap between those levels is dramatically decreased. From the experimental determination of the $B\left(E 2 ; 5 / 2^{-} \rightarrow 3 / 2^{-}\right)$ values it has become clear that the nature of the first $5 / 2^{-}$

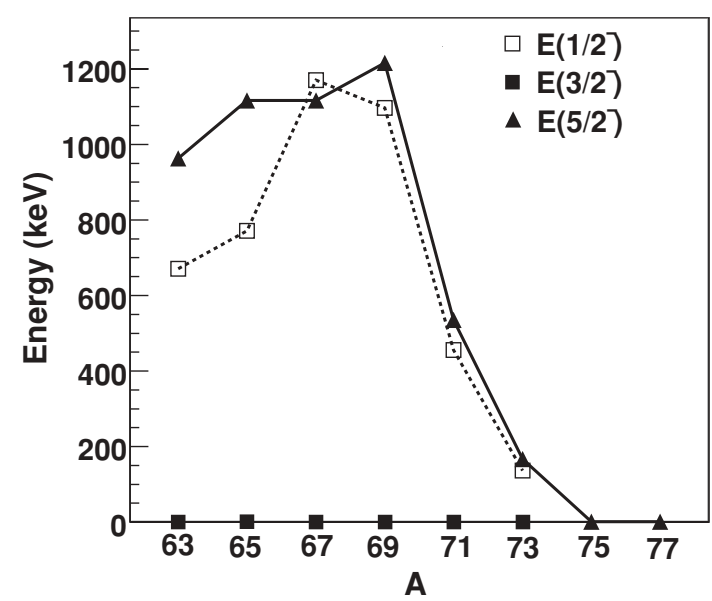

FIG. 8. Systematics of the energies of the first excited $1 / 2^{-}, 5 / 2^{-}$, and $3 / 2^{-}$states in ${ }^{63-77} \mathrm{Cu}$ (based on Refs. $[9,14,37]$ and references therein).

state in the odd $\mathrm{Cu}$ isotopes changes from a core-coupled state in the $N<40$ isotopes to a single particle state in the $N \geqslant 40$ isotopes. The single-particle character of the $5 / 2^{-}$ state is established from ${ }^{69} \mathrm{Cu}$ onward [9]. The lowering of the $5 / 2^{-}$state is strong enough to become the ground state in ${ }^{75} \mathrm{Cu}$ [37]. The observed sharp drop in energy of the $5 / 2^{-}$level, known also as monopole migration, is believed to be caused by the attractive proton-neutron $j_{>}-j_{<}$tensor force [38] (see also Refs. [9,39]).

The low-energy level structure of the neutron-rich oddmass $\mathrm{Zn}$ nuclei is dominated by the $p_{3 / 2}, f_{5 / 2}, p_{1 / 2}$, and $g_{9 / 2}$ neutron orbitals. The gradual lowering in energy of the unique parity state $g_{9 / 2}$ as a function of increasing neutron number leads from ${ }^{69} \mathrm{Zn}$ on to isomerism. In fact, from there on the states based on the $p_{1 / 2}$ and on the $g_{9 / 2}$ orbitals lie within $500 \mathrm{keV}$ of each other [22]. The situation is different in ${ }^{77} \mathrm{Zn}$ because the $\left(1 / 2^{-}\right)$state has risen up to $772 \mathrm{keV}$ above the $\left(7 / 2^{+}\right)$ground state. The positive parity levels assigned as $7 / 2^{+}$and $9 / 2^{+}$can be produced by quasiparticle couplings of the $\left(\nu g_{9 / 2}\right)^{-3}$ configuration. A similar system where three quasiparticles in the $\pi g_{9 / 2}$ orbit lead to a low-lying $7 / 2^{+}$state has been also observed in the Ag isotopes where three holes in the $\pi g_{9 / 2}$ shell result in a similar ordering of the $7 / 2^{+}$ and $9 / 2^{+}$levels. The $1 / 2^{-}$isomeric state can be interpreted as a $v p_{1 / 2}$ single-particle state. Coulomb-excitation and laserspectroscopy studies for the odd-mass $\mathrm{Zn}$ isotopes that can be performed in the future as well as the results of the recently performed laser spectroscopy experiments for the neutron-rich $\mathrm{Cu}$ isotopes [37] could contribute to a better understanding of the residual proton-neutron interaction in this mass region and the role of the $1 g_{9 / 2}$ orbital.

\section{ACKNOWLEDGMENTS}

The authors would thank B. Walters for stimulating discussions and K. L. Kratz for the use of the Mainz neutron long counter. This work was supported by The IUAP-Belgian Science Policy-BriX network (P6/23), FWO Vlaanderen (Belgium), and GOA/2004/03 (BOF-K.U.Leuven). 
[1] O. Sorlin and M.-G. Porquet, Prog. Part. Nucl. Phys. 61, 602 (2008).

[2] N. Bree et al., Phys. Rev. C 78, 047301 (2008).

[3] O. Sorlin et al., Phys. Rev. Lett. 88, 092501 (2002).

[4] R. Broda et al., Phys. Rev. Lett. 74, 868 (1995).

[5] H. Grawe and M. Lewitowicz, Nucl. Phys. A693, 116 (2001).

[6] K. Langanke, J. Terasaki, F. Nowacki, D. J. Dean, and W. Nazarewicz, Phys. Rev. C 67, 044314 (2003).

[7] C. Guénaut et al., Phys. Rev. C 75, 044303 (2007).

[8] S. Rahaman et al., Eur. Phys. J. A 34, 5 (2007).

[9] I. Stefanescu et al., Phys. Rev. Lett. 100, 112502 (2008).

[10] I. Stefanescu et al., Phys. Rev. Lett. 98, 122701 (2007).

[11] J. Van de Walle et al., Phys. Rev. Lett. 99, 142501 (2007).

[12] J. Van de Walle et al., Phys. Rev. C 79, 014309 (2009).

[13] W. F. Mueller et al., Phys. Rev. Lett. 83, 3613 (1999).

[14] S. Franchoo et al., Phys. Rev. Lett. 81, 3100 (1998).

[15] J. Van Roosbroeck et al., Phys. Rev. C 71, 054307 (2005).

[16] K.-L. Kratz et al., Astrophys. J. 403, 216 (1993).

[17] E. Kugler et al., Hyperfine Interact. 129, 23 (2000).

[18] J. Van Roosbroeck, Ph.D. thesis, IKS-Katholieke Universiteit Leuven, 2002.

[19] J.-C. Thomas et al., Phys. Rev. C 74, 054309 (2006).

[20] J. Van Roosbroeck et al., Phys. Rev. Lett. 92, 112501 (2004).

[21] J. Van Roosbroeck et al., Phys. Rev. C 69, 034313 (2004).

[22] T. Faul, Ph.D. thesis, IReS, Strasbourg, 2008.
[23] V. Mishin et al., Nucl. Instrum. Methods 73, 550 (1993).

[24] V. Fedoseyev et al., Hyperfine Interact. 127, 109 (2000).

[25] U. Köster et al., Spectrochim. Acta B 58, 1047 (2003).

[26] Oliver Arndt, Ph.D. thesis, Johannes Gutenberg Universität of Mainz, 2007.

[27] K.-L. Kratz et al., Z. Phys. A 340, 419 (1991).

[28] P. T. Hosmer et al., Phys. Rev. Lett. 94, 112501 (2005).

[29] B. Ekström et al., Phys. Scripta 34, 614 (1991).

[30] S. V. Ilyushkin et al., in Proceedings of the 4th International Conference on Fission and Properties of Neutron-Rich Nuclei, Sanibel Island, USA, 11-17 November 2007, edited by J. H. Hamilton, A. V. Ramayya, and H. K. Carter (World Scientific Publishing Co., 2007), p. 687.

[31] J. A. Winger et al., Phys. Rev. Lett. 102, 142502 (2009).

[32] A. R. Farhan and B. Singh, Nucl. Data Sheets 81, 417 (1997).

[33] P. L. Reeder and R. A. Warner, Nucl. Instrum. Methods 180, 173 (1981).

[34] G. Audi, A.-H. Wapstra, and C. Thibault, Nucl. Phys. A729, 337 (2009).

[35] J. Hakala et al., Phys. Rev. Lett. 101, 052502 (2008).

[36] B. Pfeiffer and K.-L. Kratz, Prog. Nucl. Energy 41, 39 (2002).

[37] U. Köster et al. (to be published).

[38] T. Otsuka, T. Suzuki, R. Fujimoto, H. Grawe, and Y. Akaishi, Phys. Rev. Lett. 95, 232502 (2005).

[39] S. Franchoo et al., Phys. Rev. C 64, 054308 (2001). 